\title{
Moulinet pour la détermination de la vitesse et de la direction des courants fluviaux ou marins
}

Dans le domaine des constructions et travaux hydrauliques, l'étude des courants, fluviaux et marins, au point de vue direction comme au point de vue vitesse à une très grande importance et différents constructeurs se sont appliqués à étudicr et à construire pour les intéressés des instruments répondant aux conditions d'emploi, parfois difficiles, qui se présentent dans ce genre de mesure.

Parmi les appareils ainsi réalisés, il faut citer le moulinet avec indicateur de direction étudié et construit par la maison Ott, bien connue dans le domaine de l'hydrométrie.

La vitesse et la direction du courant, au point où est immergè le moulinet, sont transmises sur le bateau par l'intermédiaire

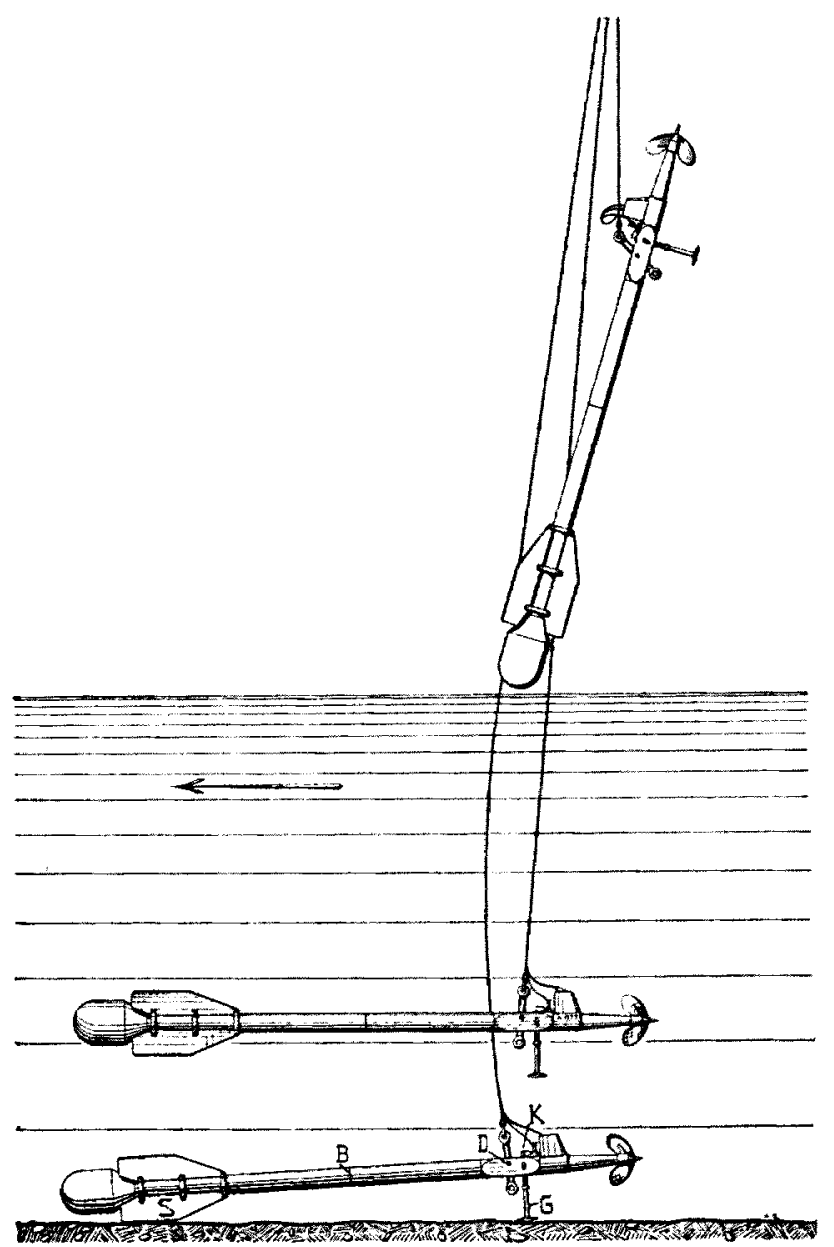

Fig. 1 déterminée à $2 \mathrm{~mm}$. près par seconde; ainsi, pour une vilesse de $1 \mathrm{~m}$. par seconde, l'crreur relative est de $1 / 500$, pour unc vitesse de 3 m.; 1/1500. Les directions sont déterminées à deux degrés près.

La profondeur maxima à laquelle peut êlre immergé l'instrument est de cent mètres, ce qui est très suffisant dans la plupart des cas.

L'ensemble de l'instrument a l'aspect d'un moulinet dil à suspension flexible. La figure 1 indique la manière dont se comporte un tel type d'appareil dans l'eau et en dehors de l'eau. Ies ailettes du gouvernail peuvent être déplacées le long du corps du moulinet pour assurer l'horizontalite parfaite en cours d'immersion. Un tel moulinel se compose de trois parlies:

$1^{\circ}$ La partic avant, constiluée par le moulinet proprement dit.

$2^{\circ}$ La partie médiane avec le dispositif de suspension articulé et portant, s'il y a lieu, la suspension du poids de lestage, muni d'un contact électrique, destiné à déceler le moment où linstrument arrive sur le fond.

30 Le gouvernail qui tient igalement lieu de flotteur-tquilibreur.

Un tel agencement a fait ses preuves depuis longtemps; il est très maniable, s'oriente rapidement et avec precision dans le courant, son démontage et son emballage s'effectuent d'une manière simple.

L'appareil dont il s'agil ici bénéficie de ces avantages. Il diffère seulement du moulinet représenlé figure 1 par le remplacement de la partie médiane, laquelle ne porte aucun organe de mesure, par une chambre étanche contenant le disposilif trans-

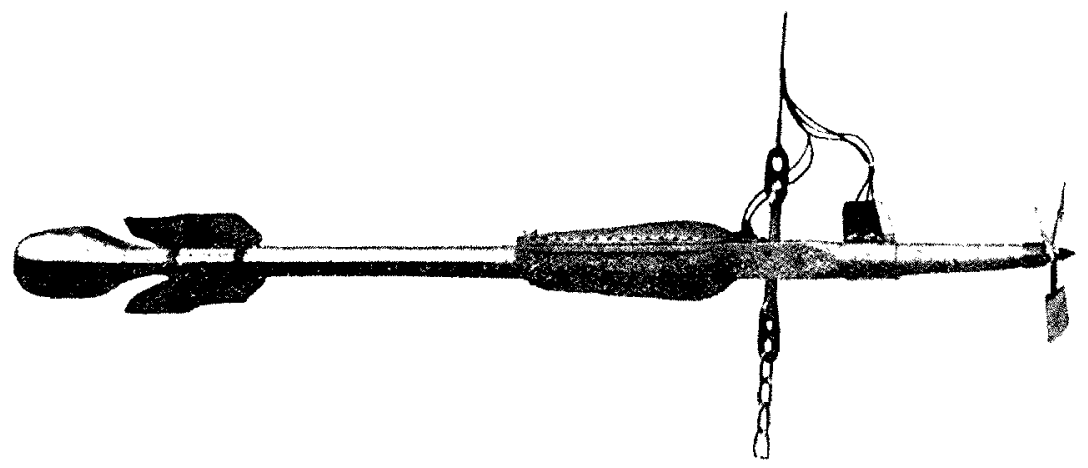

Fig. 2

metteur de direction. l'ensemble du moulinet est représenté figure 2. La résistance du moulinet au courant esl réduile au minimum par suite du profil de l'ensemble ce qui evite une trop grande inclinaison du câble porteur.

Voici la description des principales parlies de cet instrument et de ses accessoires :

Moulinel. - Il est du type Mensing Ott avec conlact en boîte 
du récepteur, ce qui dégage la roue dentéc $C^{\prime}$ de son cliquet F:' et ramène, au moyen d'un ressort, l'aiguille au O. En mème temps, l'armature de l'électro-aimant $H$ se trouve dégagé el le circuit électrique fermé en $a$. Les électro-aimants D' et D) entrent alors en jeu el, traversés par des courants dont la succession est réglée par l'interrupteur $K$, font tourner les roues dentés d'une façon synchrone. Quand la roue $\mathrm{C}$ a atteint l'aiguille aimantée, les deux électro-aimants $\mathrm{H}^{\prime}$ et $\mathrm{H}$ entrent igalement en fonctionnement; le premier interrompt te circuit en a, lautre ramène le mécanisme du transmetteur dans sa position initiale. L'aiguille du récepteur demeure dans sa position finale jusqu'a ce que, une nouvelle opération devant ère effectuée, on la ramène au zéro. La durée d'une opéralion est proportionnelle à l'angle cherché et s'élève, tout au plus, le mouvement d'horlogerie dommant quatre contacts par seconde, à 45 secondes. En ce qui concerne la graduation du cadran, nous ferons remarquer qu'elle est établie suivant la façon no" male, du Nord à l'Est, et donne la direction du point où va le courant.

Il faut noter que l'appareil continuera à fournir des indications certaines, mème dans le cas d'un defaut d'isolement (aucquel cas laide d'un relais a été prévue à cet effet) puisqu'il emploie seulement les interruptions du courant sans que la constance de celui-ci et son intensité jouent un rôle important dans le fonctionnement.

Poids de lestage. Contact de fond. - Quand on veut operer à de très grandes profondeurs, il faut, pour éviter la trop grande inclinaison du câble supportant le moulinet, surcharger celui-ci. Pour cela, on suspend à la partie inférieure de la pièce pivolanl autour de l'axe $\mathrm{D}$ (fig, 1), une surcharge de poids ct de forme déterminés, appelée "poids de lestage". Le poids est muni d'un contact électrique permettant de déceler, au moyen d'une somerie, le moment où l'instrument touche le fond.

Treuil. - La figure 5 représente le treuil utilisé pour les mesures à profondeur moyenne. La circonférence du tambour est de 1 mètre; pour une couche de câble ( 8 millimètres de diamètre), la capacité d'enroulement est de 30 mètres, et le métrage déroulé peut ètre lu sur un cadran. Il est possible de laisser le câble se clérouler de lui-même; un régulateur de vitesse à palettes intervient alors. Les contacts circulaires montés sur le lambour assurent la transmission des indications domnées par le moulinet.
Alin de determinet exactement la position en prolondeur du moulinet, meme quand le cable de suspension est plus ou moins fortement cintré par la pression de l'eau, le treuil peut ête muni d'un disposilif special de mesure de profondeur; le monlinet est alors relie, outre le câble de suspension, à un fil d'arier

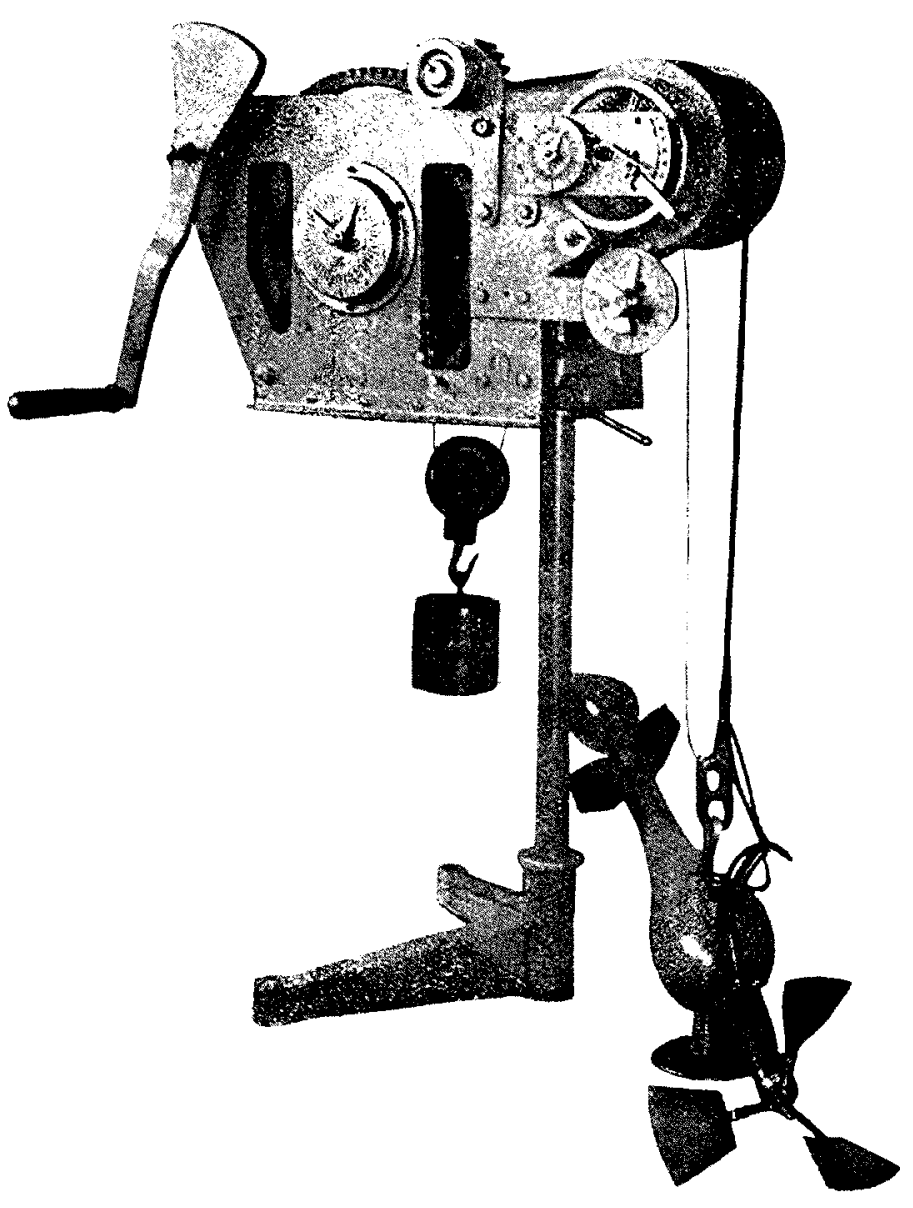

Fig. 5

de 0,6 millimètres de diamelre, a tension aulomalique, dont la longueur et l'inclinaison sur la verlicale sont mesures au moyen d'un compteur spécial. I ce produit de la longueur mesurée par le cosinus de l'angle d'inclinaison dome la distance verlicale du moulinet à son point de suspension. 